\title{
An Iterative Maximum-Likelihood Polychromatic Algorithm for CT
}

\author{
Bruno De Man, Johan Nuyts*, Patrick Dupont, Guy Marchal, and Paul Suetens
}

\begin{abstract}
A new iterative maximum-likelihood reconstruction algorithm for $\mathrm{X}$-ray computed tomography is presented. The algorithm prevents beam hardening artifacts by incorporating a polychromatic acquisition model. The continuous spectrum of the $\mathrm{X}$-ray tube is modeled as a number of discrete energies. The energy dependence of the attenuation is taken into account by decomposing the linear attenuation coefficient into a photoelectric component and a Compton scatter component. The relative weight of these components is constrained based on prior material assumptions.

Excellent results are obtained for simulations and for phantom measurements. Beam-hardening artifacts are effectively eliminated. The relation with existing algorithms is discussed. The results confirm that improving the acquisition model assumed by the reconstruction algorithm results in reduced artifacts. Preliminary results indicate that metal artifact reduction is a very promising application for this new algorithm.
\end{abstract}

Index Terms-Beam-hardening correction, computed tomography, iterative reconstruction, maximum likelihood.

\section{INTRODUCTION}

$\mathbf{S}$ OON after the invention of computed tomography (CT) [1] in 1972, reconstruction by filtered backprojection (FBP), which was developed for astrophysical applications [2], was introduced. Until today, FBP remains the most widely used reconstruction method in CT. FBP is based on the assumption that every pixel can be characterized by a single parameter $\mu$, the linear attenuation coefficient, and that the logarithm of the measurement is the line integral of $\mu$. In reality, an X-ray tube emits a continuous spectrum, and the attenuation in every pixel is energy-dependent. When a polychromatic X-ray beam passes through matter, low-energy photons are preferentially absorbed, and the beam gradually becomes harder, i.e., its mean energy increases. The harder a beam, the less it is further attenuated. Therefore, the total attenuation is no longer a linear function of

Manuscript received August 18, 2000; revised July 27, 2001. This work was supported by the Flemish Fund for Scientific Research (FWO) under Grant G.0106.98. The Associate Editor responsible for coordinating the review of this paper and recommending its publication was C. Crawford. Asterisk indicates corresponding author.

B. De Man and P. Suetens are with Medical Image Computing, RadiologyESAT/PSI, University Hospital Gasthuisberg, B-3000 Leuven, Belgium (e-mail bruno.deman@crd.ge.com).

*J. Nuyts is with the Department of Nuclear Medicine, University Hospital Gasthuisberg, B-3000 Leuven, Belgium (e-mail: Johan.Nuyts@uz.kuleuven.ac.be).

P. Dupont is with the Department of Nuclear Medicine, University Hospital Gasthuisberg, B-3000 Leuven, Belgium. He is also with the Flemish Fund for Scientific Research.

G. Marchal is with the Department of Radiology, University Hospital Gasthuisberg, B-3000 Leuven, Belgium.

Publisher Item Identifier S 0278-0062(01)09301-6. absorber thickness. Neglecting this effect leads to various wellknown beam-hardening artifacts such as cupping, the apical artifact, streaks, and flares [3]-[14].

Many different solutions for beam hardening are found in the literature:

A first approach is to limit the amount of beam hardening by physically pre-filtering the X-ray beams [4], [15].

A second approach is to correct the measurements based on certain material assumptions. The method of water correction or linearization correction assumes that all substances in the scanning plane have the same energy dependence and corrects the measurements prior to reconstruction [4], [15]-[17]. The so-called postreconstruction methods [18]-[27] make a first reconstruction, from which a rough material distribution is estimated. This material distribution allows to estimate the measurement error, based on either simulations or calibration measurements. A new reconstruction is then calculated from the corrected measurements. This process can be repeated several times (see, also, Section II-C).

A third approach is the dual energy approach [7], [16], [28]-[32]. Here, the linear attenuation coefficient is decomposed into a photoelectric and a Compton scatter component. A drawback of this approach is that it requires either two scans at different tube voltages or special detectors with two different energy windows.

Other approaches use image processing to remove beam-hardening streaks from the reconstructed images [33], [34]. An overview of beam-hardening correction approaches is given in [35].

In previous work [14] we have determined the major causes of metal artifacts. We also have shown [36] that improving the accuracy of the acquisition model assumed by the reconstruction process results in reduced artifacts. The work presented in this paper introduces a polychromatic acquisition model. A new algorithm - iterative maximum-likelihood (ML) polychromatic algorithm for CT (IMPACT)—is presented.

\section{THEORY}

IMPACT is an extension of the ML algorithm for transmission tomography (ML-TR) [36]-[38]. Section II-A treats ML-TR, Section II-B gives the derivation of IMPACT, and Section II-C describes our implementation of the postreconstruction method, used for comparison with IMPACT.

\section{A. $M L-T R$}

The basic idea is: given a set of transmission measurements $\left\{y_{i}\right\}_{i=1}^{I}$, find the distribution of linear attenuation coefficients 
$\left\{\mu_{j}\right\}_{j=1}^{J}$ that maximizes the log-likelihood (logarithm of the likelihood) [39]-[41]

$$
\mathrm{L}=\sum_{i=1}^{I}\left(y_{i} \cdot \ln \left(\hat{y}_{i}\right)-\hat{y}_{i}\right)
$$

where $\hat{y}_{i}$ is the expected number of photons detected along projection line $i$ given the current reconstruction $\left\{\mu_{j}\right\} . y_{i}$ is assumed to be a Poisson realization of $\hat{y}_{i}$. A simple acquisition model for transmission tomography is given by

$$
\hat{y}_{i}=b_{i} \cdot \exp \left(-\sum_{j=1}^{J} l_{i j} \mu_{j}\right)
$$

where $b_{i}$ is the number of photons that would be detected in the absence of absorber (blank scan) and $l_{i j}$ is the effective intersection length of projection line $i$ with pixel $j . b_{i}$ is measured by a calibration scan. The projection $\sum l_{i j} \mu_{j}$ is implemented as in [42]: for a projection line $i$ that is more vertical than horizontal $\left(\alpha \in\left[-45^{\circ} ; 45^{\circ}\right]\right.$ or $\alpha \in\left[135^{\circ} ; 225^{\circ}\right]$, where $\alpha$ is the angle between the projection line and the $y$ axis), calculate the intersection of projection line $i$ with each row, perform linear interpolation between the two adjacent columns, sum the interpolated pixel values for all rows, and multiply this sum with the intersection length of ray $i$ with one single row. For all other projection lines $(|\alpha| \in] 45^{\circ} ; 135^{\circ}[)$, do the same for all columns. Using this projection method, $l_{i j}$ becomes the product of the interpolation coefficient and the row (or column) intersection length.

An update step for maximizing (1) is given in [36]-[38]

$$
\Delta \mu_{j}=-\frac{\frac{\partial \mathrm{L}}{\partial \mu_{j}}(\vec{\mu})}{\sum_{h=1}^{J} \frac{\partial^{2} \mathrm{~L}}{\partial \mu_{j} \partial \mu_{h}}(\vec{\mu})} .
$$

Substituting (1) in (3) results in the ML-TR algorithm

$$
\mu_{j}^{n+1}=\mu_{j}^{n}+\frac{\sum_{i=1}^{I} l_{i j} \cdot\left(\hat{y}_{i}-y_{i}\right)}{\sum_{i=1}^{I} l_{i j} \cdot\left[\sum_{h=1}^{J} l_{i h}\right] \cdot \hat{y}_{i}}
$$

where $n$ is the iteration number. Fessler et al. [43] have derived a class of algorithms that monotonically increase the likelihood. Algorithm (4) is a member of that class (by choosing $\alpha_{i j}=$ $a_{i j} / \sum_{h=1}^{J} a_{i h}$ and $r_{i}=0$ in [43, Section IV-A]). In [43], $\hat{y}_{i}$ is approximated by $y_{i}$ in the denominator. The advantage is that one can then precompute the denominator prior to iterating. In the neighborhood of the optimum, both formulas are equivalent. If however the initial estimate is far from the optimal solution, the approximation leads to slower convergence. We have not applied this approximation.

\section{B. IMPACT}

A more general acquisition model than (2) is given by

$$
\hat{y}_{i}=\sum_{k=1}^{K} b_{i k} \cdot \exp \left(-\sum_{j=1}^{J} l_{i j} \mu_{j k}\right)
$$

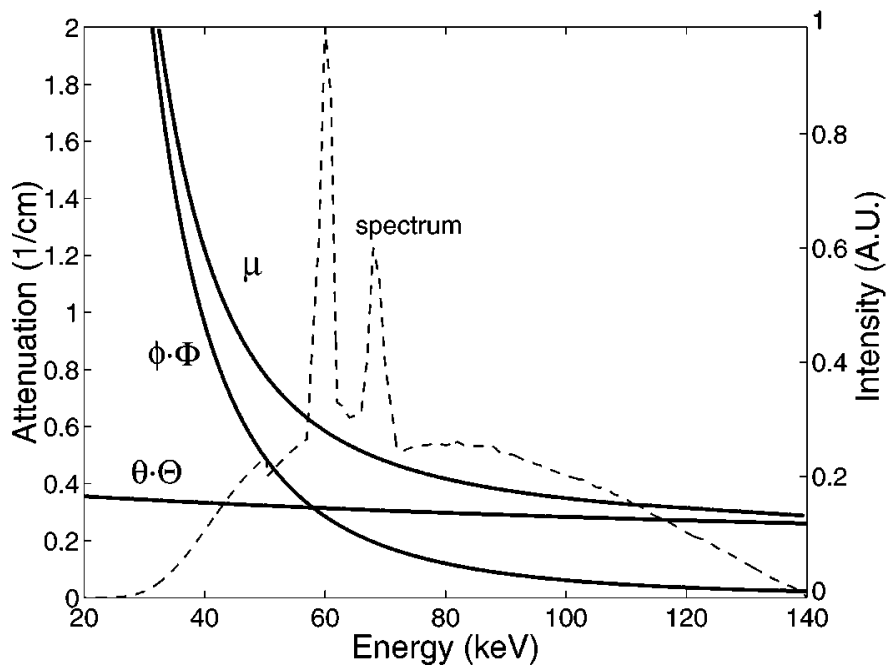

Fig. 1. Decomposition of the linear attenuation coefficient $\mu$ of bone into a photoelectric component and a Compton scatter component. The dashed line represents a simulated spectrum $b_{i k}$ (normalized) provided by Siemens.

where

$k \quad$ energy index;

$K$ total number of energies;

$\mu_{j k} \quad$ linear attenuation coefficient in pixel $j$ at energy $k$;

$b_{i k} \quad$ total energy that would be detected by detector $i$ in the absence of absorber for incident photons of energy $E_{k}$.

$b_{i k}$ is given by

$$
b_{i k}=I_{i k} \cdot S_{k} \cdot E_{k}
$$

where

$I_{i k} \quad$ emitted source spectrum (number of photons);

$S_{k} \quad$ detector sensitivity (dimensionless);

$E_{k} \quad$ photon energy (keV).

The third factor is introduced because CT uses energy-counting detectors, compared to photon-counting detectors in nuclear medicine. Consequently, the measured quantity is not a number of photons but an energy, and it will not strictly follow a Poisson distribution. This fact is usually ignored, because the Poisson model is still a good approximation (see [44] and [45]). Although in this work $b_{i k}$ is chosen independent of $i$, it could also be exploited to incorporate source fluctuations and the effect of a bow-tie filter. The number of unknowns $\mu_{j k}$ in (5) is $K \times J$, compared to $J$ in (2). This higher number of degrees of freedom (DOFs) leads to poor convergence. Hence, constraints must be introduced.

For any particular substance, the energy-dependent linear attenuation coefficient $\mu(E)$ can be approximated by a linear combination of a number of basic functions

$$
\mu(E)=a_{1} f_{1}(E)+a_{2} f_{2}(E)+\cdots+a_{n} f_{n}(E) .
$$

A good set of basic functions are the energy dependence $\Phi(E)$ of the photoelectric effect and the energy dependence $\Theta(E)$ of Compton scatter (see [17], [29]). This results in a decomposition of $\mu(E)$ into a photoelectric component and a Compton scatter component

$$
\mu(E)=\phi \cdot \Phi(E)+\theta \cdot \Theta(E) .
$$


TABLE I

PHOTOELECTRIC COEFFICIENT $\phi$, COMPTON COEFFICIENT $\theta$, AND MONOCHROMATIC LINEAR ATTENUATION COEFFICIENT $\mu_{70 \mathrm{kcV}}$ FOR A NUMBER OF COMMON SUBSTANCES

\begin{tabular}{l|l|l|l}
\hline Substances & $\theta(1 / \mathrm{cm})$ & $\phi(1 / \mathrm{cm})$ & $\mu_{70 \mathrm{keV}}(1 / \mathrm{cm})$ \\
\hline air & 0.0002 & $1.7 \mathrm{e}-05$ & 0.0002 \\
\hline fat & 0.1631 & 0.0080 & 0.1717 \\
\hline soft tissue & 0.1777 & 0.0148 & 0.1935 \\
\hline blood & 0.1778 & 0.0154 & 0.1942 \\
\hline breast & 0.1818 & 0.0118 & 0.1945 \\
\hline water & 0.1793 & 0.0144 & 0.1946 \\
\hline brain & 0.1818 & 0.0155 & 0.2022 \\
\hline muscle & 0.1793 & 0.0155 & 0.2032 \\
\hline lung & 0.1857 & 0.0158 & 0.2036 \\
\hline ovary & 0.1867 & 0.0156 & 0.2038 \\
\hline plexiglas & 0.2072 & 0.0107 & 0.2187 \\
\hline bone & 0.3109 & 0.1757 & 0.4974 \\
\hline $\mathrm{Al}$ & 0.4274 & 0.2125 & 0.6523 \\
\hline $\mathrm{Ti}$ & 0.7189 & 1.8201 & 2.6530 \\
\hline $\mathrm{Fe}$ & 1.3904 & 5.32734 & 7.0748 \\
\hline
\end{tabular}

Fig. 1 illustrates this for the case of bone. We call $\phi$ the photoelectric coefficient and $\theta$ the Compton coefficient. The energy dependence of the photoelectric effect is approximated by

$$
\Phi(E)=\frac{1 / E^{3}}{1 / E_{0}^{3}}
$$

where $E_{0}$ is a reference energy (e.g., $E_{0}=70 \mathrm{keV}$ ). This is a good approximation except for substances that have a $K$-edge in the used spectral range, such as lead or glass [46]. The energy dependence of Compton scatter is approximated by

$$
\Theta(E)=\frac{f_{\mathrm{KN}}(E)}{f_{\mathrm{KN}}\left(E_{0}\right)}
$$

where $f_{\mathrm{KN}}$ is the Klein-Nishina function, given by

$$
\begin{aligned}
f_{\mathrm{KN}}(E)= & \frac{1+\alpha}{\alpha^{2}} \cdot\left(\frac{2 \cdot(1+\alpha)}{1+2 \alpha}-\frac{\ln (1+2 \alpha)}{\alpha}\right) \\
& +\frac{\ln (1+2 \alpha)}{2 \alpha}-\frac{1+3 \alpha}{(1+2 \alpha)^{2}}
\end{aligned}
$$

with $\alpha=\mathrm{E} / 511 \mathrm{keV}$.

For any substance with known $\mu(E)$ (data from http://physics.nist.gov/PhysRefData/ based on [47] were used), $\phi$ and $\theta$ are calculated by applying a least squares fit to (8) and using the analytic formulations of $\Phi(E)$ and $\Theta(E)$ [see (9)-(11)]. In practice this fit is performed after discretization into $K$ energy levels. From (8)-(10), it can be seen that $\phi$ and $\theta$ actually represent the photoelectric part and the Compton scatter part of the attenuation at $E_{0}=70 \mathrm{keV}$. The calculated values of $\phi$ and $\theta$ and the values of $\mu_{70 \mathrm{keV}}$ are shown in Table I for some common substances.

Discretization of (8) gives

$$
\mu_{j k}=\phi_{j} \cdot \Phi_{k}+\theta_{j} \cdot \Theta_{k} .
$$

$\Phi_{k}$ and $\Theta_{k}$ represent the energy dependence of $\mu_{j k}$ and are known, dimensionless functions. $\phi_{j}$ and $\theta_{j}$ represent the material dependence and have dimension $\mathrm{cm}^{-1}$. The acquisition model of (5) becomes

$$
\hat{y}_{i}=\sum_{k=1}^{K} b_{i k} \cdot \exp \left(-\sum_{j=1}^{J} l_{i j} \cdot\left[\phi_{j} \cdot \Phi_{k}+\theta_{j} \cdot \Theta_{k}\right]\right) \text {. }
$$

The number of unknowns $\left(\phi_{j}\right.$ and $\left.\theta_{j}\right)$ is now $2 J$.

Fig. 2(a) shows a plot of $\phi$ versus $\theta$, and Fig. 2(b) is a zoomed plot for the dashed region. Most substances lie in the neighborhood of the piecewise-linear $\phi-\theta$-curve defined by the set of base substances [air, water, bone, iron] (solid line). We now assume that all substances lie $o n$ this $\phi-\theta$-curve. This assumption implies that the energy dependence of the attenuation is required to be a linear combination of the energy dependences of two adjacent base substances. Fig. 2(c) shows a plot of the monochromatic attenuation $\mu_{70 \mathrm{keV}}$ versus $\phi$ and $\theta$, and Fig. 2(d) is a zoomed plot for the dashed region. Again, the set of base substances define a $\mu-\phi$-curve and a $\mu-\theta$-curve and all substances are assumed to lie on them. For a given $\mu_{70 \mathrm{keV}}$, these functions unambiguously determine the values of $\phi$ and $\theta$. This means that $\phi_{j}$ and $\theta_{j}$ in (13) can be substituted by the functions $\phi\left(\mu_{j}\right)$ and $\theta\left(\mu_{j}\right)$, resulting in the following acquisition model:

$$
\hat{y}_{i}=\sum_{k=1}^{K} b_{i k} \cdot \exp \left(-\sum_{j=1}^{J} l_{i j}\left[\phi\left(\mu_{j}\right) \cdot \Phi_{k}+\theta\left(\mu_{j}\right) \cdot \Theta_{k}\right]\right)
$$

where $\phi\left(\mu_{j}\right)$ and $\theta\left(\mu_{j}\right)$ are known functions of $\mu_{j}$, and $\mu_{j}$ now represents the monochromatic linear attenuation coefficient at $70 \mathrm{keV}$ in pixel $j$. The number of unknowns $\mu_{j}$ in (14) is now $J$. This equation is implemented more efficiently by bringing $\Phi_{k}$ and $\Theta_{k}$ outside the summation over $j$ (projection)

$$
\hat{y}_{i}=\sum_{k=1}^{K} b_{i k} \cdot \exp \left(-\Phi_{k} \sum_{j=1}^{J} l_{i j} \phi\left(\mu_{j}\right)-\Theta_{k} \sum_{j=1}^{J} l_{i j} \theta\left(\mu_{j}\right)\right)
$$

resulting in two projections instead of $K$. Substituting (15) in (1) and applying (3) results in the IMPACT algorithm

$$
\mu_{j}^{n+1}=\mu_{j}^{n}+\Delta \mu_{j}^{n}
$$

where

$$
\begin{aligned}
\Delta \mu_{j}^{n}= & \frac{\phi_{j}^{\prime} \cdot \sum_{i=1}^{I} l_{i j} e_{i} Y_{i}^{\Phi}+\theta_{j}^{\prime} \cdot \sum_{i=1}^{I} l_{i j} e_{i} Y_{i}^{\Theta}}{\phi_{j}^{\prime} \cdot \sum_{i=1}^{I} l_{i j} M_{i}+\theta_{j}^{\prime} \cdot \sum_{i=1}^{I} l_{i j} N_{i}} \\
M_{i}= & \left(\sum_{h=1}^{J} l_{i h} \phi_{h}^{\prime}\right) \cdot\left(Y_{i}^{\Phi \Phi} \cdot e_{i}+\frac{y_{i} \cdot\left(Y_{i}^{\Phi}\right)^{2}}{\hat{y}_{i}^{2}}\right) \\
& +\left(\sum_{h=1}^{J} l_{i h} \theta_{h}^{\prime}\right) \cdot\left(Y_{i}^{\Phi \Theta} \cdot e_{i}+\frac{y_{i} \cdot Y_{i}^{\Phi} \cdot Y_{i}^{\Theta}}{\hat{y}_{i}^{2}}\right) \\
N_{i}= & \left(\sum_{h=1}^{J} l_{i h} \phi_{h}^{\prime}\right) \cdot\left(Y_{i}^{\Phi \Theta} \cdot e_{i}+\frac{y_{i} \cdot Y_{i}^{\Phi} \cdot Y_{i}^{\Theta}}{\hat{y}_{i}^{2}}\right) \\
& +\left(\sum_{h=1}^{J} l_{i h} \theta_{h}^{\prime}\right) \cdot\left(Y_{i}^{\Theta \Theta} \cdot e_{i}+\frac{y_{i} \cdot\left(Y_{i}^{\Theta}\right)^{2}}{\hat{y}_{i}^{2}}\right) \\
e_{i}= & 1-\frac{y_{i}}{\hat{y}_{i}} \\
\phi_{j}^{\prime}= & \left.\left(\frac{\partial \phi}{\partial \mu}\right)\right|_{\mu_{j}^{n}}
\end{aligned}
$$




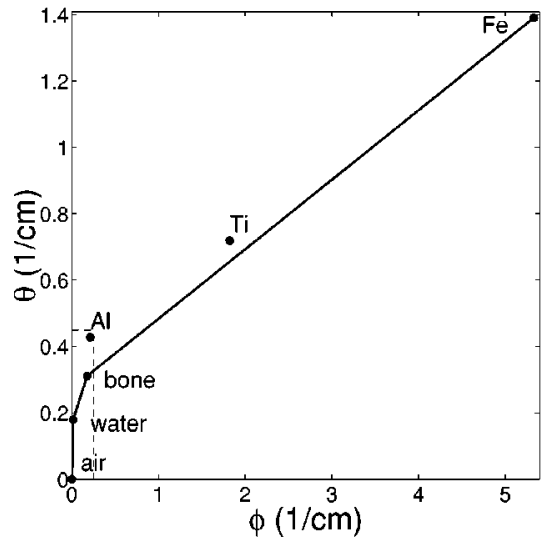

(a)

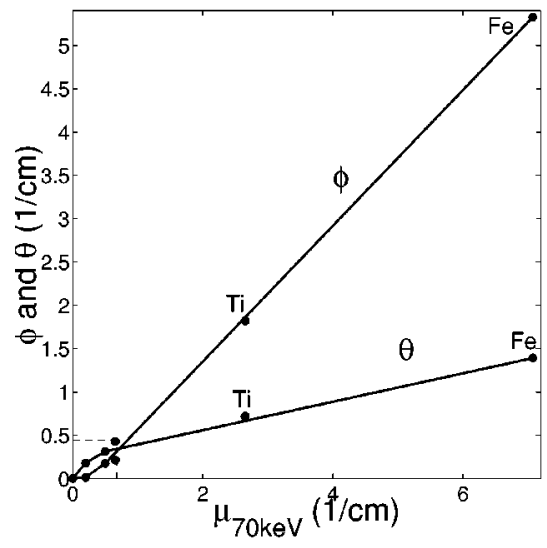

(c)

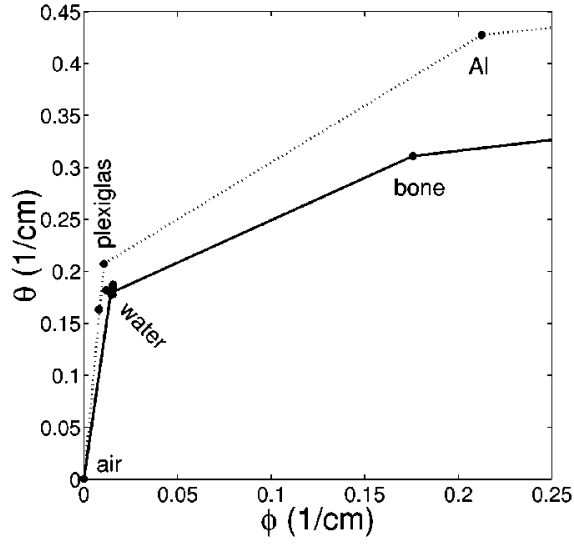

(b)

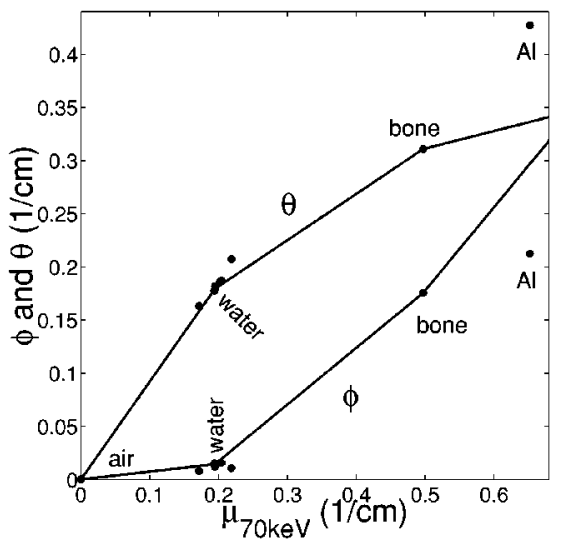

(d)

Fig. 2. (a) Photoelectric coefficient $\phi$ versus Compton coefficient $\theta$ for the set of base substances [air, water, bone, iron]. (b) Same as (a) but zoomed to the dashed region and including the other substances from Table I. (c) Monochromatic linear attenuation coefficient $\mu_{70 \mathrm{kcV}}$ versus $\phi$ and $\theta$ for the set of base substances [air, water, bone, iron]. (d) Same as (c) but zoomed to the dashed region and including the other substances from Table I.

$$
\begin{aligned}
\theta_{j}^{\prime} & =\left.\left(\frac{\partial \theta}{\partial \mu}\right)\right|_{\mu_{j}^{n}} \\
Y_{i}^{\Phi} & =\sum_{k=1}^{K} \Phi_{k} \cdot \hat{y}_{i k} \\
Y_{i}^{\Theta} & =\sum_{k=1}^{K} \Theta_{k} \cdot \hat{y}_{i k} \\
Y_{i}^{\Phi \Phi} & =\sum_{k=1}^{K} \Phi_{k} \cdot \Phi_{k} \cdot \hat{y}_{i k} \\
Y_{i}^{\Phi \Theta} & =\sum_{k=1}^{K} \Phi_{k} \cdot \Theta_{k} \cdot \hat{y}_{i k} \\
Y_{i}^{\Theta \Theta} & =\sum_{k=1}^{K} \Theta_{k} \cdot \Theta_{k} \cdot \hat{y}_{i k} \\
\hat{y}_{i k} & =b_{i k} \cdot \exp \left(-\Phi_{k} \sum_{j=1}^{J} l_{i j} \phi\left(\mu_{j}^{n}\right)-\Theta_{k} \sum_{j=1}^{J} l_{i j} \theta\left(\mu_{j}^{n}\right)\right) .
\end{aligned}
$$

Intermediate results are given in the Appendix. At the points of inflection, $\phi_{j}^{\prime}$ and $\theta_{j}^{\prime}$ are defined as

$$
\begin{aligned}
& \phi_{j}^{\prime}=\frac{1}{2} \lim _{\epsilon \rightarrow 0}\left[\left(\frac{\partial \phi}{\partial \mu}\right)\left|\mu_{j}^{n-\epsilon}+\left(\frac{\partial \phi}{\partial \mu}\right)\right|_{\mu_{j}^{n}+\epsilon}\right] \\
& \theta_{j}^{\prime}=\frac{1}{2} \lim _{\epsilon \rightarrow 0}\left[\left(\frac{\partial \theta}{\partial \mu}\right)\left|\mu_{j}^{n-\epsilon}+\left(\frac{\partial \theta}{\partial \mu}\right)\right|_{\mu_{j}^{n}+\epsilon}\right] .
\end{aligned}
$$

Finally, the result is convolved with a Gaussian smoothing kernel with standard deviation $\sigma$ to suppress Gibbs overshoots at sharp edges (cf. [48]).

The resulting image represents the monochromatic linear attenuation coefficient at $70 \mathrm{keV}$. Applying the functions $\phi\left(\mu_{j}\right)$ and $\theta\left(\mu_{j}\right)$ to these images yields the photoelectric and Compton scatter images at $70 \mathrm{keV}$. Using (8), reconstructions at other energies (even outside the used spectrum) are obtained.

Every iteration requires four projections (projection of $\phi, \theta$, $\phi^{\prime}$, and $\theta^{\prime}$ ), and four backprojections (backprojection of $e_{i} Y_{i}^{\Phi}$, $e_{i} Y_{i}^{\Theta}, M_{i}$, and $\left.N_{i}\right)$. 


\section{Postreconstruction Approach}

Our implementation of the postreconstruction approach is essentially the version of Fuchs et al. [27]. All other methods [18]-[26] are either limited to two or three substances, or apply some extra approximations because of limited computational power at the time they were invented. Similar to IMPACT, a set of base substances (e.g., [air, water, bone, iron]) is chosen, assuming that every pixel contains a mixture of two adjacent base substances. An initial FBP-reconstruction is calculated from the original sinogram. For every substance, an image containing the substance concentrations is segmented and each image is projected into a substance sinogram. Every substance sinogram pixel contains the total amount of that substance along the corresponding projection line. For every sinogram pixel, the monochromatic projections (at $E_{0}$ ) and the polychromatic projections (based on a $K$-energy spectrum) are calculated. The difference between the monochromatic and the polychromatic sinogram is added to the original sinogram and a new FBP-reconstruction is obtained. This process is repeated $N$ times. In the remainder, we refer to this method as iterative beam-hardening correction (IBHC). In our implementation, computation time increases linearly with the number of substances considered but is still much lower than for ML or related iterative algorithms.

\section{METHODS}

\section{A. Simulations}

Our two-dimensional CT-simulator is described and validated in [14]. Simulations are performed at increased sampling, taking into account the continuous rotation of the tube-detector unit and the finite width of the focal spot and the detector elements. Phantoms are defined as a superposition of a number of basic objects, each with its own shape, composition, size, position and resolution.

We defined four circular water phantoms, each with a diameter of $19 \mathrm{~cm}$. Phantom 1 consists of water only and is used to study the cupping artifact in the presence of one single substance. Phantom 2 contains four circular bone regions $(\phi=3$ $\mathrm{cm}$ and $1 \mathrm{~cm}$ ) and is used to study beam hardening in the presence of at least two different substances. Phantom 3 contains eight circular regions $(\phi=3 \mathrm{~cm})$ of fat, aluminum, plexiglas, lung, brain, bone, blood, and soft tissue (in clockwise order and starting from the top). This is used to investigate how critical is the set of base substances assumed by IMPACT and IBHC. Phantom 4 is similar to phantom 2, but the two smallest cylindrical inserts are now made of iron $(\phi=1 \mathrm{~cm})$. This phantom was designed to study beam hardening in the presence of metal objects.

Simulation parameters were adjusted to the Siemens Somatom Plus4 CT-scanner with fixed focal spot and a 0.75-s rotation. Both monochromatic and polychromatic (by summing photons at 50 discrete energy levels) simulations were performed. A simulated spectrum $b_{i k}$ provided by Siemens and corresponding to a nominal tube voltage of $140 \mathrm{kV}$ was used (Fig. 1). Generally, no noise or scatter was included, because we want to focus on beam-hardening artifacts. For phantom 4 , an extra simulation was performed with noise $\left(b_{i}=10^{5}\right.$

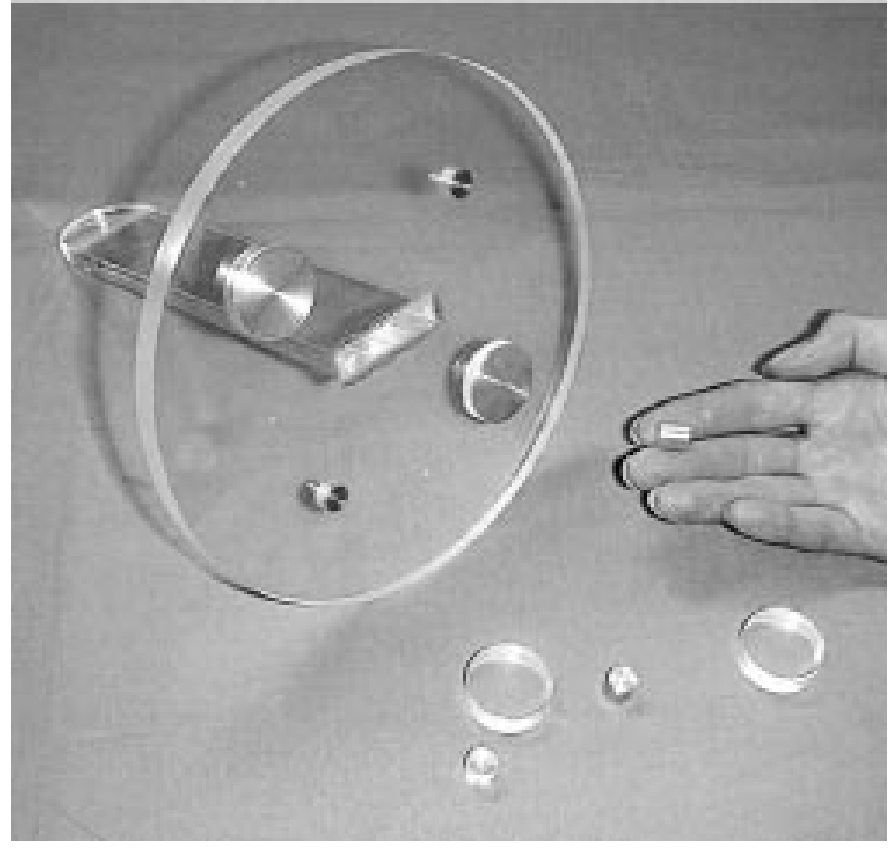

Fig. 3. Picture of the plexiglas phantom.

photons) to investigate the importance of the noise model in the presence of metal objects.

\section{B. Measurements}

Three phantoms were scanned on a Siemens Somatom Plus4 CT-scanner. Phantom 5 is a circular plexiglas phantom (Fig. 3) with thickness $1 \mathrm{~cm}$, a diameter of $19 \mathrm{~cm}$, and containing four aluminum cylinders $(\phi=1 \mathrm{~cm}$ and $3 \mathrm{~cm})$. This phantom is comparable to software phantom 2 . We chose plexiglas and aluminum instead of water and bone for practical reasons. This phantom is used to study beam hardening in the absence of metal objects. Phantom 6 is similar to phantom 5, but the two smallest cylindrical inserts are now made of iron $(\phi=1 \mathrm{~cm})$. This phantom is comparable to software phantom 4 and it is used to study beam hardening in the presence of metal objects. Phantom 7 is a skull phantom (borrowed from Siemens): a human skull filled and surrounded by plexiglas. The skull phantom is used to study beam hardening on a clinically more relevant phantom.

We used sequential scan mode with fixed focal spot, a nominal slice thickness of $1.0 \mathrm{~mm}$, a 0.75 -s rotation, a nominal tube voltage of $140 \mathrm{kV}$ or $120 \mathrm{kV}$ (skull), and a tube current of $206 \mathrm{~mA}$ or $240 \mathrm{~mA}$ (skull). The raw data were transferred to a PC for further processing using our own reconstruction software.

\section{Reconstruction}

All simulations and measurements were reconstructed with:

- FBP: using a Hamming window with a cutoff frequency equal to half the maximum frequency.

- ML-TR: using 50 iterations of 100 subsets +50 iterations of ten subsets (cf. [49]), $\sigma=0.9$ pixels. $\sigma$ is the standard deviation of the Gaussian smoothing kernel and was chosen so that ML-TR and IMPACT resulted in images 


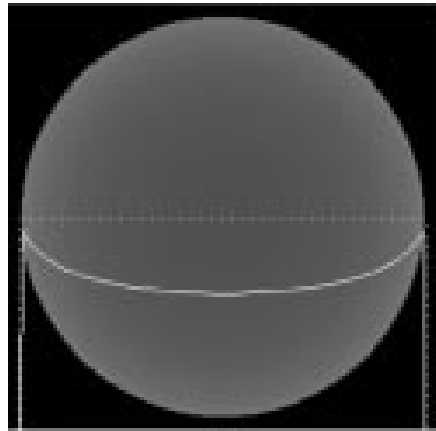

(a)

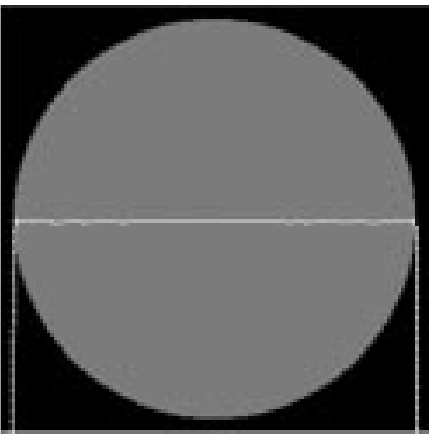

(b)

Fig. 4. Phantom 1 (simulation). (a) FBP. (b) IMPACT. The white curves represent the middle rows of the respective images. The dotted white curves represent FBP of the monochromatic simulation.

with the same degree of edge smoothing as the FBP and IBHC reconstructions.

- IMPACT: using 50 iterations of 100 subsets +50 iterations of ten subsets, $\sigma=0.9$ pixels, $K=20, E_{0}=70 \mathrm{keV}$. Unless stated differently, [air, water, bone, iron] was used as set of base substances for the simulations [solid line in Fig. 2(b)] and [air, plexiglas, aluminum, iron] was used as set of base substances for the measurements [dotted line in Fig. 2(b)].

- IBHC: $N=5, K=20, E_{0}=70 \mathrm{keV}$. The same sets of base substances were used as for IMPACT.

A simulated spectrum $b_{i k}$ provided by Siemens (Fig. 1) was used for both IMPACT and IBHC.

\section{RESULTS}

\section{A. Simulations}

All simulation results are shown using a windowing interval $\mu$ $=[0.175 ; 0.215] \mathrm{cm}^{-1}$ (centered at $\left.\mu_{\text {water }}\right)$, which corresponds to a window of $200 \mathrm{HU}$ and a level of $0 \mathrm{HU}$. The images are 20 $\mathrm{cm} \times 20 \mathrm{~cm}$ and $256 \times 256$ pixels.

Fig. 4 shows the reconstructions of phantom 1 . The white curves represent the middle rows of the respective images. The dotted white curves represent FBP of the monochromatic simulation, which is used as reference. For the polychromatic simulation, both FBP Fig. 4(a) and ML-TR (not shown) result in a substantial amount of cupping: the values are depressed toward the center of the object. This cupping is entirely eliminated both with IMPACT Fig. 4(b) and IBHC (not shown). The perfect overlap of the white curves in Fig. 4(b) shows that the images are also quantitatively correct.

Fig. 5 shows the reconstructions of phantom 2. FBP of the monochromatic simulation Fig. 5(a) is used as reference. For the polychromatic simulation, both FBP Fig. 5(b) and ML-TR (not shown) exhibit severe artifacts: the bone inserts are connected by dark streaks. These streaks are effectively eliminated both with IMPACT Fig. 5(c) and IBHC Fig. 5(d). IMPACT requires at least 50 iterations for convergence, IBHC only two.

Fig. 6 shows the reconstructions of phantom 3. FBP of the monochromatic simulation Fig. 6(a) is used as reference. For the polychromatic simulation, both FBP Fig. 6(b) and ML-TR

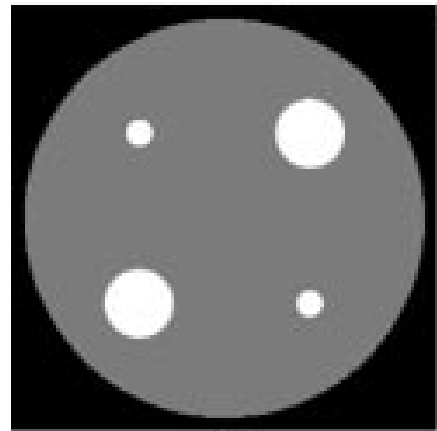

(a)

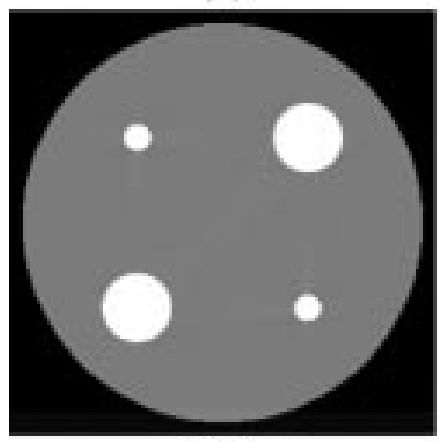

(c)

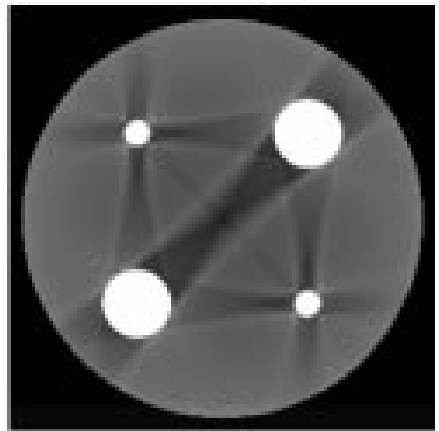

(b)

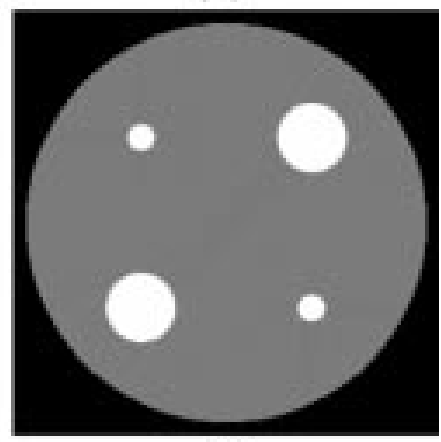

(d)
Fig. 5. Phantom 2 (simulation). (a) FBP (mono). (b) FBP. (c) IMPACT. (d) IBHC.

(not shown) exhibit severe artifacts: the bone and aluminum inserts are connected by dark streaks. These streaks are reduced but not eliminated both with IMPACT Fig. 6(c) and with IBHC Fig. 6(d). Using an extended set of base substances [air, water, plexiglas, bone, aluminum, iron] beam-hardening artifacts are effectively eliminated both with IMPACT Fig. 6(e) and IBHC Fig. 6(f). It appears that aluminum and plexiglas are too far separated from the $\phi-\theta$-curve defined by [air, water, bone, iron], while all human tissues (fat, lung, brain, blood, soft tissue, ...) are sufficiently close to water.

Fig. 7 shows the reconstructions of phantom 4. FBP of the monochromatic simulation Fig. 7(a) is used as reference. It shows some streak artifacts connecting the metal and bone inserts. These streaks are not due to beam hardening, but to other effects such as the nonlinear partial volume effect [50] and aliasing [51]-[53]. For the polychromatic simulation, both FBP Fig. 7(b) and ML-TR (not shown) exhibit severe artifacts: the bone and metal inserts are connected by dark streaks. The streaks that were present in Fig. 7(b) but not in Fig. 7(a) are eliminated both with IMPACT Fig. 7(c) and IBHC Fig. 7(e). The remaining streaks in Fig. 7(c) and (e) are similar to the streaks in Fig. 7(a), which indicates that the beam-hardening artifacts are effectively eliminated. Fig. 7(d) and (f) shows the IMPACT and IBHC results for the simulation with noise $\left(b_{i}=10^{5}\right.$ photons). In both images, beam-hardening artifacts are partially masked by noise artifacts. Clearly, the IMPACT reconstruction benefits from the correct noise model resulting in less severe noise artifacts. This is shown quantitatively by calculating the standard deviation in an excentric circular water region. This resulted in a standard deviation of $0.0028 \mathrm{~cm}^{-1}$ for IMPACT and $0.0039 \mathrm{~cm}^{-1}$ for IBHC. 


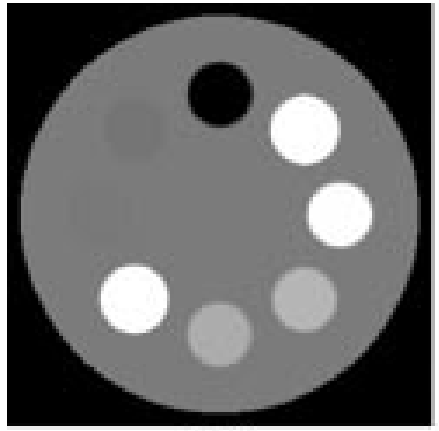

(a)

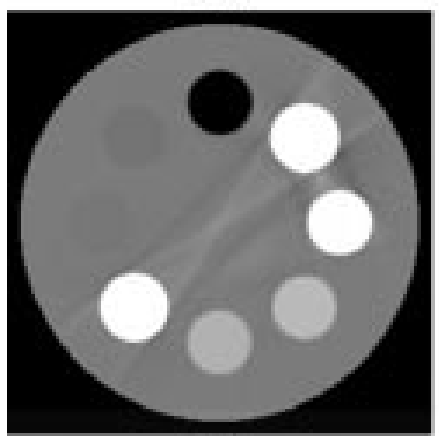

(c)

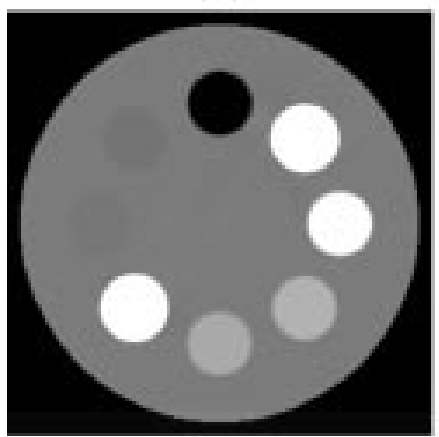

(e)

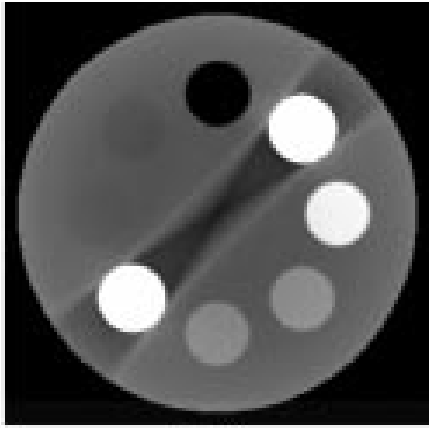

(b)

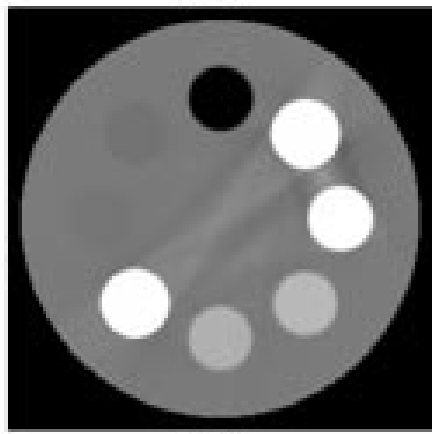

(d)

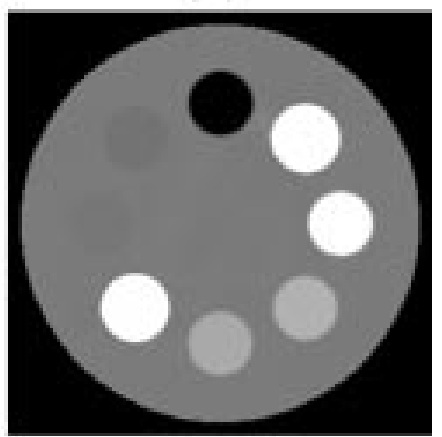

(f)

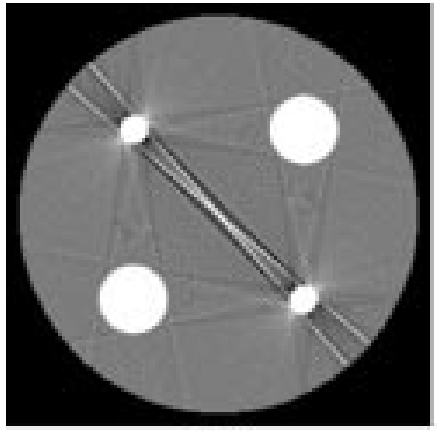

(a)

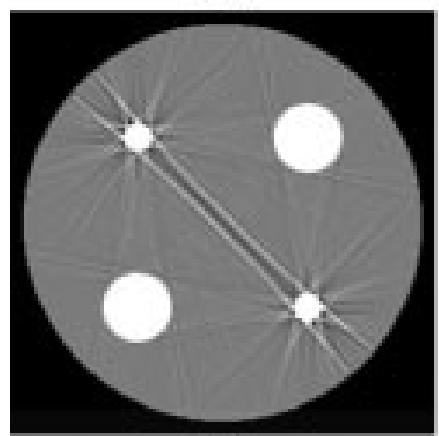

(c)

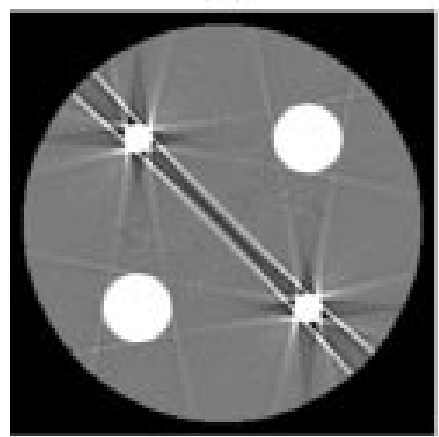

(e)

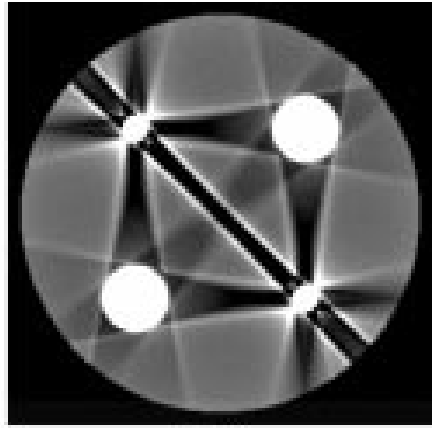

(b)

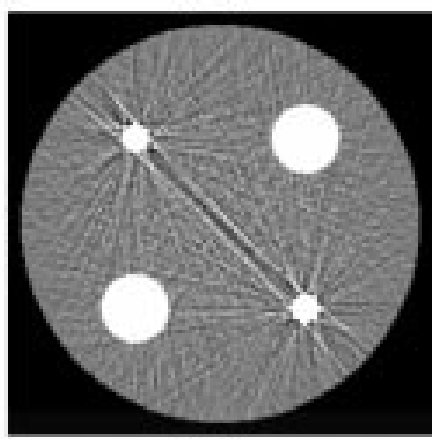

(d)

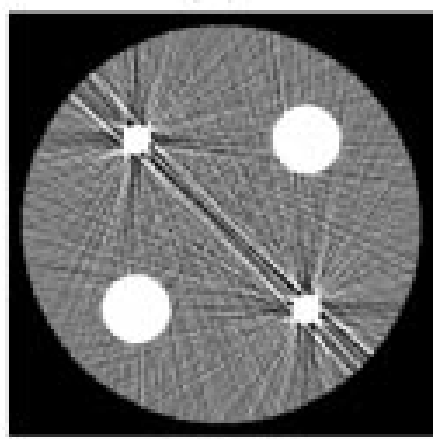

(f)

Fig. 6. Phantom 3 (simulation). (a) FBP (mono). (b) FBP. (c) IMPACT. (d) IBHC. (e) IMPACT with extended set of base substances. (f) IBHC with extended set of base substances.

\section{B. Measurements}

All measurement results are shown using a windowing interval $\mu=[0.20 ; 0.24] \mathrm{cm}^{-1}$ (centered at $\mu_{\text {plexiglas }}$ ), which corresponds to a window of $200 \mathrm{HU}$ and a level of $125 \mathrm{HU}$. The images are $20 \mathrm{~cm} \times 20 \mathrm{~cm}$ and $256 \times 256$ pixels.

The left column of Fig. 8 shows the reconstructions of phantom 5. The FBP reconstruction Fig. 8(a) exhibits a number of dark beam-hardening streaks. The streaks are strongly reduced both with IMPACT Fig. 8(c) and IBHC Fig. 8(e).

The right column of Fig. 8 shows the reconstructions of phantom 6. The FBP reconstruction Fig. 8(b) exhibits severe streak artifacts. The dark streaks are reduced both with IMPACT Fig. 8(d) and IBHC Fig. 8(f). Comparison with phantom 4 indicates that the remaining streaks are mainly due to other effects, such as noise, scatter, the nonlinear partial volume effect, and aliasing. The remaining dark streak in Fig. 8(f)—probably due to scatter-is more prominent than in Fig. 8(d). This also suggests that IMPACT benefits from its correct noise model,

Fig. 7. Phantom 4 (simulation). (a) FBP (mono). (b) FBP. (c) IMPACT. (d) IMPACT (with noise). (e) IBHC. (f) IBHC (with noise).

which makes IMPACT robust against errors corresponding to strongly attenuated measurements.

Fig. 9 shows the reconstructions of phantom 7. The FBP reconstruction Fig. 9(a) exhibits dark beam-hardening streaks. These streaks are effectively eliminated with IMPACT Fig. 9(b) and IBHC Fig. 9(d). [air, plexiglas, bone, iron] was chosen as set of base substances. Subtracting IMPACT from FBP shows where the correction for beam-hardening artifacts is most prominent Fig. 9(c). It also shows a strong reduction of the background noise. Note also that the bone regions are dark. This is mainly due to the fact that the IMPACT reconstruction represents values at $70 \mathrm{keV}$, while the FBP reconstruction represents averages over the entire spectrum.

\section{DISCUSSION}

We have derived a new algorithm that combines three existing approaches. First, it is an ML iterative algorithm, which allows to use a correct noise model and an acquisition model that can 


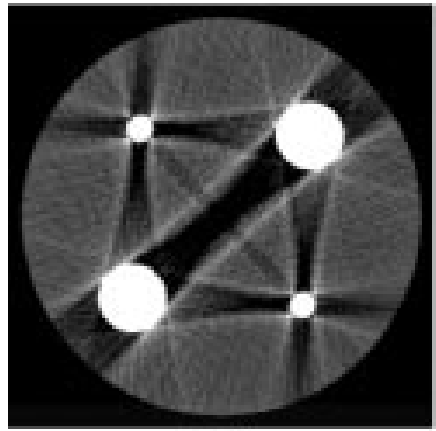

(a)

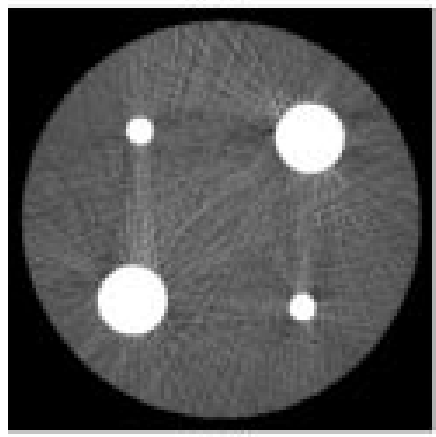

(c)

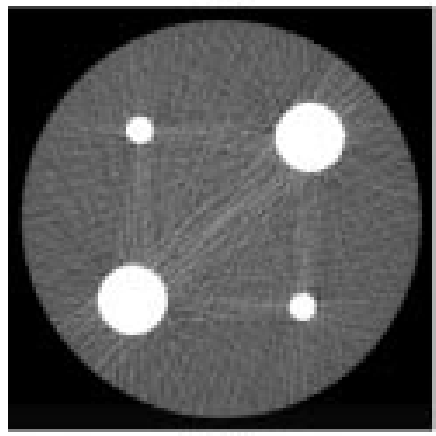

(e)

Fig. 8. Phantom 5 and phantom 6 (measurements). (a)-(b) FBP. (c)-(d) IMPACT. (e)-(f) IBHC.

be extended to other sources of artifacts, in this case to polychromaticity. Second, similar to the dual energy approach, the attenuation coefficient is decomposed into a photoelectric and a scatter component. This is a natural decomposition that strongly reduces the number of DOFs without loss of generality. Third, similar to IBHC, the domain of the linear attenuation coefficient is confined to mixtures of known substances.

The quality of the IMPACT reconstructions is, in the absence of metal objects, not better than the IBHC reconstructions: both result in effective beam-hardening correction. The results of phantom 4 and 5 indicate that, in the presence of metal objects, IMPACT benefits from its correct noise model resulting in less severe noise and other artifacts. A more extended evaluation of the algorithm with respect to noise, spatial resolution, quantitative accuracy, and degree of artifacts is planned after including models for scatter and the nonlinear partial volume effect.

One important parameter is the assumed set of base substances. The simulations of phantom 3 show that this set is not

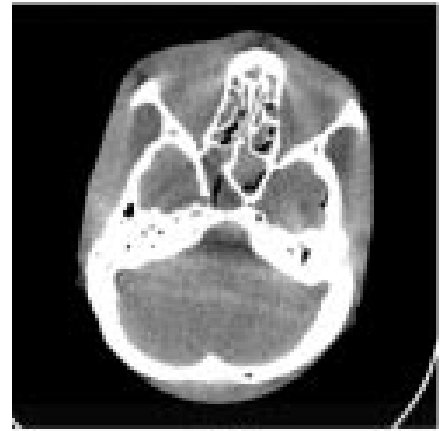

(a)

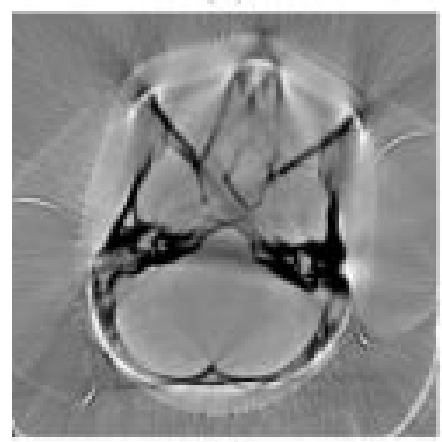

(c)

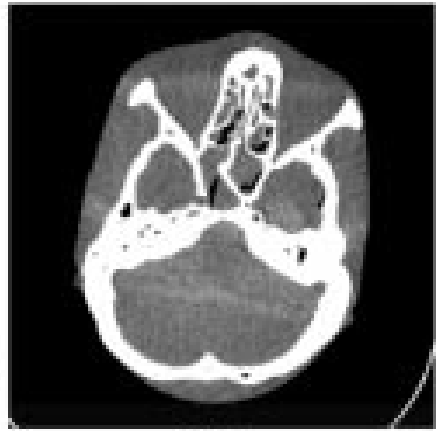

(b)

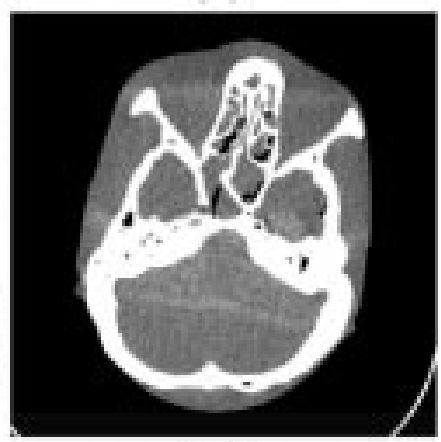

(d)
Fig. 9. Phantom 7 (measurement). (a) FBP. (b) IMPACT. (c) Subtraction of IMPACT and FBP. (d) IBHC.

very critical provided that the substances in the scanning plane are not too far separated from the assumed $\phi-\theta$-relationship. It is advisable to include in the $\phi-\theta$-relationship all substances that are known to be present in the scanning plane. For routine clinical use, we suggest to use the set of base substances [air, soft tissue, bone, teeth]. In case other materials (e.g., titanium, amalgam, ...) are known to be present, these should be added as well. Unlike in IBHC, the number of these substances can become large without increase in algorithmic complexity or computational load. This also suggests a way of increasing the speed of IBHC: decomposing the linear attenuation coefficient into a photoelectric and Compton scatter components-just like in IMPACT - amounts in two projections per iteration instead of a projection per iteration for each modeled substance. Note that materials with a $\mathrm{K}$-edge in the linear attenuation coefficient may require to extend the algorithm to a third component. There is one situation where the proposed model might not be optimal, namely in the presence of two materials with the same effective attenuation but with different photoelectric and Compton scatter components.

Another important parameter is the used spectrum. As shown by the results, the simulated spectrum provided by the manufacturer results in effective beam-hardening correction. The number of energy levels $K$ can be increased with only marginal increase in computation time. The results show that using $K=20$ is sufficient for an effective beam-hardening correction. For larger objects it could be opportune to take into account the nonuniformity of the spectrum due to the bow-tie filter.

The main drawback of IMPACT is the high computation time inherent to ML iterative reconstruction. Therefore, it is probably advisable to use IBHC as long as no metal streak artifacts 
are present. As a first-order approximation, we can write reconstruction time as

$$
t \sim \frac{\text { nriter } \cdot \text { nrdet } \cdot \text { nrviews } \cdot \text { imsize } \cdot \text { nrslices } \cdot \text { complexity }}{\text { CPU }}
$$

where

$\begin{array}{ll}\text { nriter } & \text { number of iterations; } \\ \text { nrdet } & \text { number of detector elements; } \\ \text { nrviews } & \text { number of views; } \\ \text { imsize } & \begin{array}{l}\text { number of rows or columns in the reconstruc- } \\ \text { tion; }\end{array} \\ \text { nrslices } & \text { number of slices; } \\ \text { complexity } & \text { number of (back) projections per iteration; } \\ \text { CPU } & \text { CPU-speed. }\end{array}$

The complexity is 1 for FBP, 3 for ML-TR (or 2 if the denominator is kept constant), eight for IMPACT, and $(1+$ number of materials $+1 /$ nriter) for IBHC. Typically, using a Pentium III-800, computation time is a few hours for nrdet $=768$, nrviews $=1056$, nriter $=100$, imsize $=512$, nrslices $=1$, and complexity $=8$. This may prevent IMPACT from being used in clinical routine the next few years. Until now, we have focused on a fundamental solution to the problem, rather than worrying about computation times. We are convinced that additional acceleration tricks, more efficient programming, and faster computers and hardware implementation will bring ML iterative reconstruction in $\mathrm{CT}$ within reach.

In our opinion, the main contribution of this work is the incorporation of a polychromatic model into a ML iterative reconstruction algorithm. This allows to extend existing ML approaches with beam-hardening correction. The method requires only one scan (in contrast with the dual energy approach), and both the number of discrete energies and the number of substances taken into account can be increased with only marginal increase in computation time (in contrast with IBHC). In our case, we have previously [36] shown that ML-TR is promising for metal artifact reduction, mainly thanks to the noise model, and by adding a Markov Random Field prior. We now have extended this method with a model for polychromaticity. It is confirmed that improving the acquisition model results in reduced artifacts. The algorithm can further be extended by including other effects such as scatter and the nonlinear partial volume effect in the acquisition model. A common advantage of IMPACT and IBHC is that the monochromatic reconstructions allow a direct quantitative interpretation, compared with classical images, which are averages over the used spectrum. The difference between IMPACT and other beam-hardening correction approaches (IBHC in particular) can be summarized as follows. Instead of correcting the data in order to obey certain assumptions imposed by the reconstruction algorithm, the reconstruction process is adapted to the data by incorporating a more accurate acquisition model. Polychromatic data are not transformed into monochromatic data, but the algorithm takes into account that the measurements actually are polychromatic.

\section{CONCLUSION}

We have presented an iterative ML algorithm for CT (IMPACT) that prevents beam-hardening artifacts. Excellent results were obtained on simulations and phantom measurements.
The algorithm has been compared to the postreconstruction approach, and the degree of beam-hardening correction was comparable for both algorithms. A strong reduction of computation time is required before being used routinely. Preliminary results indicate that metal artifact reduction is a very promising application for this new algorithm. Previous work has investigated the importance of the correct noise model. This paper addresses a second important cause of metal artifacts, namely beam hardening. Further research will extend the algorithm to include a prior, a model for scatter, and a model for the nonlinear partial volume effect.

\section{APPENDIX}

From (15) it follows that

$$
\frac{\partial \hat{y}_{i}}{\partial \mu_{j}}=-l_{i j}\left(\phi_{j}^{\prime} Y_{i}^{\Phi}+\theta_{j}^{\prime} Y_{i}^{\Theta}\right)
$$

and assuming $\phi_{j}^{\prime \prime} \equiv 0$ and $\theta_{j}^{\prime \prime} \equiv 0$ we have

$$
\begin{aligned}
\frac{\partial^{2} \hat{y}_{i}}{\partial \mu_{j} \partial \mu_{h}}=-l_{i j} l_{i h}( & \phi_{j}^{\prime} \phi_{h}^{\prime} Y_{i}^{\Phi \Phi}+\phi_{j} \theta_{h}^{\prime} Y_{i}^{\Phi \Theta} \\
& \left.+\theta_{j} \phi_{h}^{\prime} Y_{i}^{\Phi \Theta}+\theta_{j}^{\prime} \theta_{h}^{\prime} Y_{i}^{\Theta \Theta}\right) .
\end{aligned}
$$

From (1) it follows that

$$
\frac{\partial L}{\partial \mu_{j}}=\sum_{i=1}^{I}\left(e_{i}\right)\left(-l_{i j}\right)\left(\phi_{j}^{\prime} Y_{i}^{\Phi}+\theta_{j}^{\prime} Y_{i}^{\Theta}\right)
$$

and

$$
\begin{aligned}
\frac{\partial^{2} L}{\partial \mu_{j} \partial \mu_{h}}= & -\sum_{i=1}^{I}\left(l_{i j}\right)\left(l_{i h}\right) \\
& {\left[e_{i}\left(\phi_{j}^{\prime} \phi_{h}^{\prime} Y_{i}^{\Phi \Phi}+\phi_{j} \theta_{h}^{\prime} Y_{i}^{\Phi \Theta}+\theta_{j} \phi_{h}^{\prime} Y_{i}^{\Phi \Theta}+\theta_{j}^{\prime} \theta_{h}^{\prime} Y_{i}^{\Theta \Theta}\right)\right.} \\
& \left.\frac{y_{i}}{\hat{y}_{i}^{2}}-\left(\phi_{j}^{\prime} Y_{i}^{\Phi}+\theta_{j}^{\prime} Y_{i}^{\Theta}\right)\left(\phi_{h}^{\prime} Y_{i}^{\Phi}+\theta_{h}^{\prime} Y_{i}^{\Theta}\right)\right] .
\end{aligned}
$$

Substituting this in (3) and reordering the terms results in (16).

\section{ACKNOWLEDGMENT}

The authors would like to thank the people from Siemens Medical Systems (S. Schaller in particular) for their cooperation.

\section{REFERENCES}

[1] G. N. Hounsfield, "Computerized transverse axial scanning (tomography): Part 1. Description of system," Br. J. Radiol., vol. 46, pp. 1016-1022, Dec. 1973.

[2] R. N. Bracewell and A. C. Riddle, "Inversion of fanbeam scans in radio astronomy," Astrophys. J., vol. 150, pp. 427-434, 1967.

[3] M. Gado and M. Phelps, "The peripheral zone of increased density in cranial computed tomography," Radiology, vol. 117, pp. 71-74, Oct. 1975.

[4] R. A. Brooks and G. Di Chiro, "Beam hardening in X-ray reconstructive tomography," Phys. Med. Biol., vol. 21, no. 3, pp. 390-398, 1976.

[5] L. M. Zatz and R. E. Alvarez, "An inaccuracy in computed tomography: The energy dependence of CT values," Radiology, vol. 124, pp. 91-97, July 1977.

[6] G. Di Chiro, R. A. Brooks, L. Dubal, and E. Chew, "The apical artifact: Elevated attenuation values toward the apex of the skull," J. Comput. Assist. Tomogr., vol. 2, no. 1, pp. 65-70, Jan. 1978. 
[7] A. J. Duerinckx and A. Macovski, "Polychromatic streak artifacts in computed tomography images," J. Comput. Assist. Tomogr., vol. 2, pp. 481-487, 1978.

[8] _ - "Nonlinear polychromatic and noise artifacts in X-ray computed tomography images," J. Comput. Assist. Tomogr., vol. 3, pp. 519-526, 1979.

[9] F. Earnest, E. C. McCullough, and D. A. Frank, "Fact or artifact: An analysis of artifact in high-resolution computed tomographic scanning of the Sella," Radiology, vol. 140, pp. 109-113, July 1981.

[10] P. S. Rao and R. J. Alfidi, "The environmental density artifact: A beamhardening effect in computed tomography," Radiology, vol. 141, pp. 223-227, Oct. 1981.

[11] S. W. Young, H. H. Muller, and W. H. Marshall, "Computed tomography: Beam hardening and environmental density artifact," Radiology, vol. 148, pp. 279-283, July 1983.

[12] U. Moström and C. Ytterbergh, "Artifacts in computed tomography of the posterior fossa: A comparative phantom study," J. Comput. Assist. Tomogr., vol. 10, no. 4, pp. 560-566, 1986.

[13] C. Ruth and P. M. Joseph, "A comparison of beam-hardening artifacts in X-ray computerized tomography with gadolinium and iodine contrast agents," Med. Phys., vol. 22, no. 12, pp. 1977-1982, Dec. 1995.

[14] B. De Man, J. Nuyts, P. Dupont, G. Marchal, and P. Suetens, "Metal streak artifacts in X-ray computed tomography: A simulation study," IEEE Trans. Nucl. Sci., vol. 46, pp. 691-696, June 1999.

[15] W. D. McDavid, R. G. Waggener, W. H. Payne, and M. J. Denis, "Correction for spectral artifacts in cross-sectional reconstruction from X-rays," Med. Phys., vol. 4, no. 1, pp. 54-57, 1997.

[16] S. C. Pang and S. Genna, "Correction for X-ray polychromaticity effects on three-dimensional reconstruction," IEEE Trans. Nucl. Sci., vol. 23, pp. 623-626, Feb. 1976.

[17] G. T. Herman, "Correction for beam hardening in computed tomography," Phys. Med. Biol., vol. 24, no. 1, pp. 81-106, 1979.

[18] P. M. Joseph and R. D. Spital, "A method for correcting bone induced artifacts in computed tomography scanners," J. Comput. Assist. Tomogr., vol. 2, pp. 100-108, 1978.

[19] P. R Rüegsegger, T. Hangartner, H. U. Keller, and T. Hinderling, "Standardization of computed tomography images by means of a material-selective beam hardening correction," J. Comput. Assist. Tomogr., vol. 2, no. 2, pp. 184-188, Apr. 1978

[20] P. K. Kijewski and B. E. Bjärngard, "Correction for beam hardening in computed tomography," Med. Phys., vol. 5, no. 3, pp. 209-214, 1978.

[21] G. T. Herman, "Demonstration of beam hardening correction in computed tomography of the head," J. Comput. Assist. Tomogr, vol. 3, no. 3, pp. 373-378, June 1979

[22] O. Nalcioglu and R. Y. Lou, "Post-reconstruction method for beam hardening in computerised tomography," Phys. Med. Biol., vol. 24, no. 2, pp. 330-340, 1979

[23] G. T. Herman and S. S. Trivedi, "A comparative study of two postreconstruction beam hardening correction methods," IEEE Trans. Med. Imag., vol. MI-2, pp. 128-135, Sept. 1983.

[24] D. D. Robertson and H. K. Huang, "Quantitative bone measurements using X-ray computed tomography with second-order correction," Med. Phys., vol. 13, no. 4, pp. 474-479, 1986.

[25] J. M. Meagher, C. D. Mote, and H. B. Skinner, "CT image correction for beam hardening using simulated projection data," IEEE Trans. Nucl. Sci., vol. 37, pp. 1520-1524, Apr. 1990.

[26] P. M. Joseph and C. Ruth, "A method for simultaneous correction of spectrum hardening artifacts in CT images containing both bone and iodine," Med. Phys., vol. 24, no. 10, pp. 1629-1634, 1997.

[27] T. Fuchs, "Strahlaufhärtungskorrekturen in der computer-tomographie," Ph.D. dissertation, Institut für Medizinische Physik, Friedrich-Alexander Universität, Erlangen-Nürnberg, Germany, 1998.

[28] R. E. Alvarez and A. Macovski, "Energy-selective reconstructions in X-ray computerized tomography," Phys. Med. Biol., vol. 21, no. 5, pp. 733-744, 1976

[29] D. E. Avrin, A. Macovski, and L. M. Zatz, "Clinical application of compton and photo-electric reconstruction in computed tomography: Preliminary results," Invest. Radiol., vol. 13, no. 3, pp. 217-222, 1978.

[30] G. Christ, "Exact treatment of the dual-energy method in CT using polyenergetic X-ray spectra," Phys. Med. Biol., vol. 29, no. 12, pp. $1501-1510,1984$.
[31] A. Hemmingsson, B. Jung, and C. Ytterbergh, "Dual energy computed tomography: Simulated monoenergetic and material-selective imaging," J. Comput. Assist. Tomogr., vol. 10, no. 3, pp. 490-499, 1986.

[32] C. H. Yan, R. T. Whalen, G. S. Beaupré, S. Y. Yen, and S. Napel, "Reconstruction algorithm for polychromatic CT imaging: Application to beam hardening correction," IEEE Trans. Med. Imag., vol. 19, pp. 1-11, Jan. 2000.

[33] D. J. Mott, G. Zheng, and B. Eddleston, "The removal of a cupping artefact from brain images produced by the EMI 7070 CT scanner," $B r$. J. Radiol., vol. 58, pp. 873-880, Sept. 1985.

[34] H. Soltanian-Zadeh, J. P. Windham, and J. Soltanianzadeh, "CT artifact correction: An image processing approach,” SPIE, vol. 2710, pp. 477-485, 1996

[35] J. P. Stonestrom, R. E Alvarez, and A. Macovski, "A framework for spectral artifact corrections in X-ray CT," IEEE Trans. Biomed. Eng., vol. 28 , no. 2, pp. 128-141, Feb. 1981.

[36] B. De Man, J. Nuyts, P. Dupont, G. Marchal, and P. Suetens, "Reduction of metal streak artifacts in X-ray computed tomography using a transmission maximum a posteriori algorithm," IEEE Trans. Nucl. Sci., vol. 47, no. 3, pp. 977-981, June 2000.

[37] J. Nuyts, P. Dupont, and L. Mortelmans, "Iterative reconstruction of transmission sinograms with low signal to noise ratio," in Proc. 2nd IEEE Workshop CMP: Can We Beat the Curse of Dimensionality (Aug. 28-30, 1996). Prague, Czech Republic: Birkhäuser, 1997, pp. 237-248.

[38] J. Nuyts, B. De Man, P. Dupont, M. Defrise, P. Suetens, and L. Mortelmans, "Iterative reconstruction for helical CT: A simulation study," Phys. Med. Biol., vol. 43, pp. 729-737, 1998.

[39] A. J. Rockmore and A. Macovsky, "A maximum likelihood approach to emission image reconstruction from projections," IEEE Trans. Nucl. Sci., vol. 23, pp. 1428-1432, 1976.

[40] K. Lange and R. Carson, "EM reconstruction algorithms for emission and transmission tomography," J. Comput. Assist. Tomogr., vol. 8, no. 2, pp. 306-316, 1984.

[41] E. U. Mumcuoglu, R. Leahy, S. R. Cherry, and Z. Zhou, "Fast gradientbased methods for Bayesian reconstruction of transmission and emission PET images," IEEE Trans. Med. Imag., vol. 13, pp. 687-701, Aug. 1994.

[42] P. M. Joseph, "An improved algorithm for reprojecting rays through pixel images," IEEE Trans. Med. Imag., vol. MI-1, pp. 192-196, June 1983.

[43] J. A. Fessler, E. P. Ficaro, N. H. Clinthorne, and K. Lange, "Grouped-coordinate ascent algorithms for penalized-likelihood transmission image reconstruction," IEEE Trans. Med. Imag., vol. 16, pp. 166-175, Apr. 1997.

[44] H. Guan and R. Gordon, "Computed tomography using algebraic reconstruction techniques (ART's) with different projection access schemes: A comparison study under practical situations," Phys. Med. Biol., vol. 41, no. 9, pp. 1727-1743, 1996.

[45] J. Hsieh, "Adaptive streak artifact reduction in computed tomography resulting from excessive X-ray photon noise," Med. Phys., vol. 25, no. 11, pp. 2139-2147, 1998.

[46] I. Kaplan, Nuclear Physics. Reading, MA: Addison-Wesley, 1979.

[47] ICRU, International Commission on Radiation Units and Measurements, "Tissue substitutes in radiation dosimetry and measurement,", Bethesda, MD, Tech. Rep. 44, 1989.

[48] D. L. Snyder, M. I. Miller, L. J. Thomas, and D. G. Politte, "Noise and edge artifacts in maximum-likelihood reconstructions for emission tomography," IEEE Trans. Med. Imag., vol. MI-6, pp. 228-238, June 1987.

[49] H. M. Hudson and R. S. Larkin, "Accelerated image reconstruction using ordered subsets of projection data," IEEE Trans. Med. Imag., vol. 13, pp. 601-609, Aug. 1994.

[50] P. M. Joseph and R. D. Spital, "The exponential edge-gradient effect in X-ray computed tomography," Phys. Med. Biol., vol. 26, no. 3, pp. 473-487, 1981.

[51] R. A. Brooks, G. H. Glover, A. J. Talbert, R. L. Eisner, and F. A. DiBianca, "Aliasing: A source of streaks in computed tomograms," $J$. Comput. Assist. Tomogr., vol. 3, no. 4, pp. 511-518, 1979.

[52] C. R. Crawford and A. C. Kak, "Aliasing artifacts in computerized tomography," Appl. Opt., vol. 18, no. 21, pp. 3704-3711, 1979.

[53] C. D. Stockham, "A simulation study of aliasing in computed tomography,” Radiology, vol. 132, pp. 721-726, 1979. 